\title{
Tropical pyomyositis as a presenting feature of subclinical leukemia: a case report
}

\author{
Mitrakrishnan Rayno Navinan*, Jevon Yudhisdran, Thambyaiah Kandeepan and Aruna Kulatunga
}

\begin{abstract}
Introduction: Pyomyositis, though classically considered a tropical disease, has a variable geographic prevalence. Among the predisposing risk factors, immunodeficiency plays an important role. Pyomyositis has a tendency to mimic more commonly considered diseases, and a lack of familiarity with it is a cause of delayed diagnosis.

Case presentation: A 53-year-old South Asian man with newly diagnosed type 2 diabetes mellitus was referred to our medical unit in an advanced stage of the disease, which was complicated by sepsis and acute kidney injury. Failure of the referring unit to provide prompt treatment, as well as their delay in coming to a diagnosis, led to the patient's complicated state. Antibiotic therapy was initiated, and clinical stabilization was achieved with supportive measures. Following the patient's recovery from sepsis, his persistent leukopenia and anemia was suggestive of an underlying immunodeficiency, and a subsequent bone marrow biopsy revealed acute myeloid leukemia, M2 variant. Multi-disciplinary care was initiated by the medical, surgical and oncological teams.

Conclusion: Awareness of tropical pyomyositis is lacking. Common predisposing behaviors and conditions should always be sought and investigated. Immunosuppressive state is an important predisposing factor in the pathogenesis of pyomyositis. Early antibiotic treatment is pivotal in management, and surgical intervention, when relevant, should not be delayed. Identifying one cause should not halt the search for others, as pyomyositis may herald underlying sinister diseases.
\end{abstract}

Keywords: AML, Immunodeficiency, Leukemia, Tropical pyomyositis

\section{Introduction}

Pyomyositis is classically considered a disease of the tropics and has been known to make up $4 \%$ of all admissions in certain African nations [1]. Immunity plays a pivotal role, as it has been observed to be commoner in patients in compromised states, whether it be secondary to HIV or to non-infective states predisposing the patient to immune system impairment (for example, diabetes mellitus, rheumatologic diseases and malignancy). This phenomenon has thus caused an increased prevalence of pyomyositis, even in temperate countries [2]. In addition to impaired immunity, other risk factors include intravenous drug use and trauma that may be considered trivial [3]. Characteristically, the predominant causative organism is Staphylococcus aureus, which may even be methicillin-resistant $[4,5]$. However, in patients in immunocompromised states, organisms may show a varying

\footnotetext{
*Correspondence: rayno.navinan@gmail.com

National Hospital of Sri Lanka, Regent Street, Colombo 10, Sri Lanka
}

pattern, such as Gram-negative bacteria; Streptococcus groups B, C and G; Mycobacterium avium [4]; and many others. Pyomyositis mainly affects the major muscle groups of the limb girdles, especially the quadriceps, causing suppuration leading to abscesses $[3,6]$. The diagnosis may be difficult, as it may mimic more commonly considered diseases of the tropics, such as cellulitis, dengue, leptospirosis, viral fever and osteomyelitis [7]. This may be attributed to lack of familiarity or the absence of specific signs [3]. In this report, we present a unique case of tropical pyomyositis that heralded an underlying silent and sinister etiology masked by the presence of obvious risk factors requiring management with a multi-disciplinary approach.

\section{Case presentation}

A 53-year-old South Asian man recently diagnosed with type 2 diabetes mellitus who had been receiving treatment for 2 months with oral hypoglycemic drugs presented to our hospital with slow, insidious, progressive 
swelling of the anterior aspect of the left thigh. He was otherwise healthy and denied any high-risk behavior, smoking or alcohol consumption in the past. His presentation to us was delayed, as he initially failed to seek attention with worsening symptoms. Upon his initial admission to the hospital for investigation and management, despite persistent fever, the investigating unit failed to suspect pyomyositis. They treated the patient with intravenous cloxacillin 1g every 6 hours, suspecting cellulitis. With the development of acute confusion, the patient was presented to our medical unit for further management. The patient had no preceding history of trauma. The patient's examination revealed that he was febrile, had a high body temperature $\left(39^{\circ} \mathrm{C}\right)$ and was confused (Glasgow Coma Scale score of 11 out of 15) with acidotic breathing. His abdominal, respiratory and remaining neurologic system examination results were normal. Despite his condition, the patient's blood pressure remained within normal parameters, though tachycardia was noted. His left thigh was observed to be disproportionately swollen, warm and tense to touch.

Whole-blood analysis revealed a grossly elevated white blood cell count of $34 \times 10^{9} / \mathrm{L}$ (normal range, $4 \times 10^{9} / \mathrm{L}$ to $\left.11 \times 10^{9} / \mathrm{L}\right)$, which was predominantly neutrophilic $(86 \%)$. He had a low hemoglobin concentration of $6.8 \mathrm{~g} / \mathrm{dl}$ (normal range, $11 \mathrm{~g} / \mathrm{dl}$ to $18 \mathrm{~g} / \mathrm{dl}$ ) and a platelet count of $158 \times 10^{9} / \mathrm{L}$ (normal range, $150 \times 10^{9} / \mathrm{L}$ to $450 \times 10^{9} / \mathrm{L}$ ). His C-reactive protein concentration was elevated at $258 \mathrm{mg} / \mathrm{L}$ (normal range, $<8 \mathrm{mg} / \mathrm{L}$ ). His blood culture tests were negative repeatedly. His serum creatinine level was elevated at $140 \mu \mathrm{mol} / \mathrm{L}$ (normal range, $60 \mu \mathrm{mol} / \mathrm{L}$ to $120 \mu \mathrm{mol} / \mathrm{L}$ ) and rising, with the highest value recorded at $195 \mu \mathrm{mol} / \mathrm{L}$. Despite his rising creatinine level, his electrolytes remained normal. His coagulation profile was deranged, with an elevated international normalized ratio of 2.59 and an activated thromboplastin time of 41 seconds (normal range, 24 to 36 seconds). His serum ammonia level was elevated at $69 \mu \mathrm{mol} / \mathrm{L}$ (normal range, $26 \mu \mathrm{mol} / \mathrm{L}$ to $47 \mu \mathrm{mol} / \mathrm{L}$ ), and he had a high serum lactate dehydrogenase value of 531IU/L (normal range, 230IU/L to $460 \mathrm{IU} / \mathrm{L}$ ). His total bilirubin was elevated at $93 \mu \mathrm{mol} / \mathrm{L}$ (normal range, $5 \mu \mathrm{mol} /$ $\mathrm{L}$ to $21 \mu \mathrm{mol} / \mathrm{L}$ ) and showed a predominant indirect fraction. His alkaline phosphatase concentration was marginally elevated at 345IU/L (normal range, 98IU/L to 279IU/ $\mathrm{L}$ ), but his aspartate and alanine transaminase values were normal at 32IU/L (normal range, $<34 \mathrm{IU} / \mathrm{L}$ ) and $38 \mathrm{IU} / \mathrm{L}$ (normal range, 11IU/L to 50IU/L), respectively. His total proteins were found to be mildly reduced at $5.1 \mathrm{~g} / \mathrm{dl}$ (normal range, $6.4 \mathrm{~g} / \mathrm{dl}$ to $8.4 \mathrm{~g} / \mathrm{dl}$ ). Though marginally low, his albumin level remained predominant, with a value of $2.7 \mathrm{~g} /$ $\mathrm{dl}$ (normal range, $3.7 \mathrm{~g} / \mathrm{dl}$ to $5.4 \mathrm{~g} / \mathrm{dl}$ ). His preliminary fasting blood sugar values were found to be elevated at $215 \mathrm{mg} / \mathrm{dl}$ (normal range, less than $126 \mathrm{mg} / \mathrm{dl}$ ), reflecting poor control.
His blood picture revealed normocytic normochromic red cells, polychromatic cells, red cell fragments with toxic neutrophils and mild thrombocytopenia. These features were compatible with hemolysis secondary to sepsis. The result of retroviral screening was negative. Ultrasound imaging of the abdomen revealed a slightly hypoechoic, mildly enlarged liver with normal ducts and splenomegaly of $13.36 \mathrm{~cm}$. Magnetic resonance imaging (MRI) of the patient's left thigh and pelvis revealed a multi-loculated mass, most likely an abscess, which measured $20 \mathrm{~cm}$ in length and $8 \mathrm{~mm}$ in its widest diameter. The mass demonstrated high signal intensity in the medial aspect of the thigh on T2-weighted images (Figure 1), confirming our clinical suspicion of pyomyositis. Cultures of the drained pus were found to be sterile. However, a muscle biopsy and culture was positive for a non-lactose fermenting coliform organism (sensitive to cefoperazone and sulbactam, netilmicin and ciprofloxacin; intermediate sensitivity to ticarcillin-clavulanate; and resistance

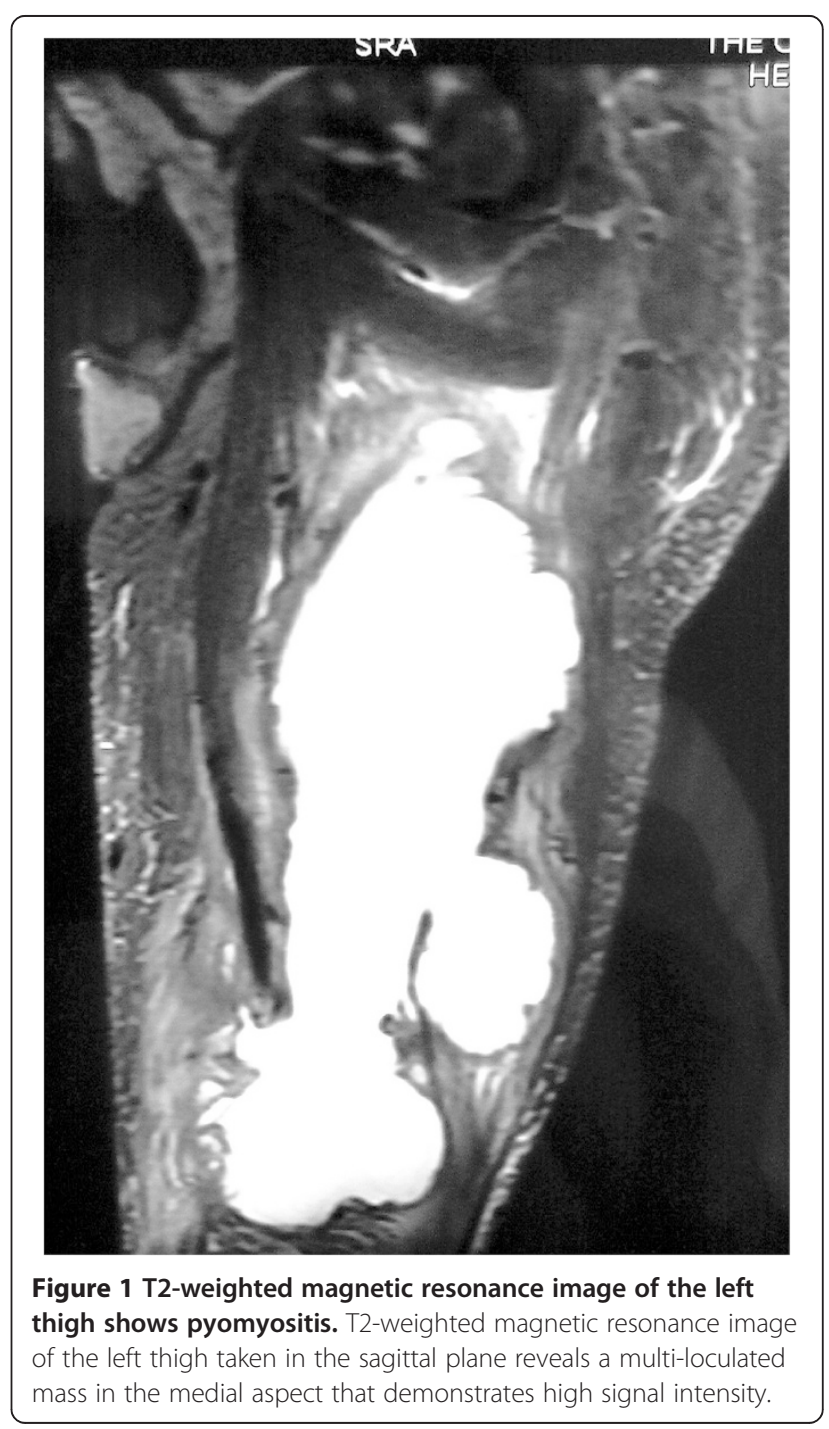


to imipenem, meropenem, amikacin, ceftazidime and gentamicin). A coagulase-negative, methicillin-resistant Staphylococcus aureus isolate was also detected, but contamination was considered likely.

Thus, the patient was initiated on intravenous cefoperazone and sulbactam 1 g every 12 hours for 2 weeks. In view of his rising serum creatinine level, we opted for one cycle of hemodialysis with strict fluid management, which resulted in resolution of his acute kidney injury. He was transfused with packed red blood cells to achieve and maintain his hemoglobin levels. He demonstrated dramatic clinical improvement, with normalization of consciousness and stabilization of his whole-blood count values to normal. After 8 days of treatment and achievement of stabilization, the significant amount of pus necessitated surgical intervention. Thus, a surgical consult was sought. The patient's loculated pus was drained, which was found to be sterile when cultured. Tight glycemic control was achieved with subcutaneous administration of soluble insulin, and the patient's capillary blood sugar values were maintained below $200 \mathrm{mg} / \mathrm{dl}$. However, following drainage and treatment with intravenous antibiotics, the development and persistence of leukopenia $\left(2.51 \times 10^{9} / \mathrm{L}\right.$ (normal range, $4 \times 10^{9} / \mathrm{L}$ to $\left.11 \times 10^{9} / \mathrm{L}\right)$ ) with lymphocytic predominance of $81 \%$ (normal range, $20 \%$ to $40 \%$ ), coupled with normocytic normochromic anemia in whole-blood analysis despite clinical improvement, prompted further investigation. On the basis of suspecting an underlying leukemia, a bone marrow biopsy was done and revealed a markedly hypercellular marrow with $22 \%$ abnormal blasts and reactive granulocytic hyperplasia. The flow cytometric and morphologic findings were compatible with acute myeloid leukemia (AML), M2 variant (AML-M2). The patient was then referred for further oncologic management.

\section{Discussion}

South Asian countries such as India and Sri Lanka are classified as tropical; however, only a few sporadic cases and case series of tropical pyomyositis have been reported [8-10]. This may be due to an overall lack of awareness and underreporting rather than the lack of prevalence. In this report, we present a case of a patient with pyomyositis who demonstrated classic etiologic predisposing factors: underlying subclinical AML with type 2 diabetes mellitus that progressed to systemic sepsis with acute kidney injury. The unique presenting manifestation of AML in our patient was pyomyositis due to secondary infection.

Immunocompromised states should always be excluded when pyomyositis is suspected [3]. Although the tropical environment by itself is a risk factor, South Asians also have a high incidence of type 2 diabetes mellitus, a wellknown cause of impaired immunity and a risk factor for pyomyositis. Our patient had type 2 diabetes mellitus. Though it was a recent diagnosis, it would have been a silent yet established predisposing etiologic factor. What made the case unique, however, was his dual pathology of AML-M2, which further enhanced the effect of his immunocompromised state. This might explain the large amount of pus with its complexity and loculation, and even the late stage of presentation. This case reemphasizes the need to carry out all relevant investigations to exclude underlying causes of immunocompromise. The identification of one predisposing cause should not stop the etiologic search for others, because underlying subclinical sinister causes may be discovered that require urgent intervention. The importance of immunocompetence and its impact on the presentation of pyomyositis is demonstrated by our present case. Hematologic malignancies are known to be associated with pyomyositis. Falagas and colleagues [11], in their comprehensive review, detailed the various malignancies that have been reported thus far. These include AML, acute lymphoblastic leukemia, acute myelomonocytic leukemia, chronic myeloid leukemia, chronic lymphocytic leukemia, myeloproliferative disease, myelodysplastic syndrome, plasma cell leukemia, myelomonocytic leukemia, multiple myeloma, non-Hodgkin's and Hodgkin's lymphoma, diffuse large B-cell lymphoma and lymphoblastic lymphoma. Though Staphylococcus aureus predominated in most of these documented cases, a variety of atypical organisms were also noted.

Treatment of pyomyositis depends on staging, which is categorized into three stages [3,6]. Stage 1 (invasive stage) is early and has relatively mild signs and symptoms, with minimal systemic signs and little or no pus. Stage 2 (suppurative stage) is an advanced stage in which most present, and the symptoms are severe due to presence of pus. Stage 3 (late stage) is the final stage, where there is systemic toxicity, dissemination of infection and organ dysfunction. Only the preliminary stage may be managed medically; the latter two require a combination of medical management with surgical intervention [3,6]. The case of our patient demonstrates the typical insidious pattern that progresses to purulent collections, also described by Patel and colleagues [12]. Though our patient was in the latter part of stage 2 when he sought treatment initially, his condition deteriorated quickly to stage 3. He was referred to us in a state of sepsis complicated by confusion and acute kidney injury and without a definitive diagnosis. After we made a confident diagnosis based on the patient's history and physical signs and affirmed by MRI, the patient was hemodialyzed and his septic state was stabilized, and he was referred back for drainage of the loculated pus collection with follow-up oncologic intervention.

Tropical pyomyositis can be a disease with multiple clinical complexities. The presence of it should alert the 
clinician to be wary of conditions that predispose individuals to such a disease. Tropical pyomyositis may be the first heralding feature of yet to be diagnosed clinical conditions that may be more sinister than the disease process itself.

\section{Conclusion}

Awareness of tropical pyomyositis is lacking among the medical fraternity, and a high degree of clinical suspicion should facilitate early diagnosis. Though antibiotic treatment is pivotal in the management of pyomyositis and needs to be initiated early surgical intervention, when deemed clinically relevant, should be sought. Immunosuppression plays a key role in the pathogenesis of pyomyositis, and a full spectrum of investigations to exclude common predisposing conditions should always be carried out. The identification of one cause should not halt the search for others, as rarely tropical pyomyositis may herald sinister diseases that are subclinical. Thus, typical risk factors and behaviors should be sought for and investigated, and, through the course of the illness, complications should be anticipated and managed promptly.

\section{Consent}

Written informed consent was obtained from the patient for publication of this case report and any accompanying images. A copy of the written consent is available for review by the Editor-in-Chief of this journal.

\section{Abbreviations}

AML: Acute myeloid leukemia; MRI: Magnetic resonance imaging.

\section{Competing interests}

The authors declare that they have no competing interests.

\section{Authors' contributions}

TK, AK, MRN and JY diagnosed the clinical scenario. MRN and AK researched and drafted the manuscript. All authors provided care for the patient. All authors read and approved the final manuscript.

\section{Authors' information}

MRN is a registrar of medicine at the National Hospital of Sri Lanka, Colombo. JY is a senior registrar in medicine at the National Hospital of Sri Lanka, Colombo. TK is a senior registrar in medicine at the National Hospital of Sri Lanka, Colombo. AK is a Consultant physician in acute medicine at the National Hospital of Sri Lanka, Colombo.

\section{Acknowledgments}

The authors would like to acknowledge the contribution of the doctors, nurses and laboratory technicians in the Departments of Radiology, Microbiology and Haematology and the general surgical units of the National Hospital of Sri Lanka for their contributions to making the diagnosis and for aiding in the management of the patient

Received: 29 July 2014 Accepted: 4 January 2015

Published online: 15 February 2015

\section{References}

1. Horn CV, Master S. Pyomyositis tropicans in Uganda. East Afr Med J. 1968:45:463-71.

2. Crum NF. Bacterial pyomyositis in the United States. Am J Med. 2004;117:420-8.
3. Chauhan S, Jain S, Varma S, Chauhan SS. Tropical pyomyositis (myositis tropicans): current perspective. Postgrad Med J. 2004:80:267-70.

4. Dunkerley GR, Older J, Onwochei B, Pazienza J. Pyomyositis. Am Fam Physician. 1996:54:565-9.

5. Ruiz ME, Yohannes S, Wladyka CG. Pyomyositis caused by methicillin-resistant Staphylococcus aureus. N Engl J Med. 2005:352:1488-9.

6. Chiedozi LC. Pyomyositis: review of 205 cases in 112 patients. Am J Surg 1979;137:255-9.

7. George P, Bendigeri M. Tropical pyomyositis-an emerging multi-disciplinary emergency. Trop Med Surg. 2013;1:114.

8. Gambhir IS, Singh DS, Gupta SS, Gupta PR, Kumar M. Tropical pyomyositis in India: a clinico-histopathological study. J Trop Med Hyg. 1992;95:42-6.

9. Malhotra P, Singh S, Sud A, Kumari S. Tropical pyomyositis: experience of a tertiary care hospital in north-west India. J Assoc Physicians India. 2000:48:1057-9.

10. Ralapanawa DMPUK, Wijeratne RVARTS, Mangaleshwaran S. A case of Tropical Pyomyositis. Annual Academic Sessions of Kandy Society of Medicine. Kandy Society of Medicine: Kandy; 2007.

11. Falagas ME, Rafailidis PI, Kapaskelis A, Peppas G. Pyomyositis associated with hematological malignancy: case report and review of the literature. Int J Infect Dis. 2008;12:120-5.

12. Patel SR, Olenginski TP, Perruquet JL, Harrington TM. Pyomyositis: clinical features and predisposing conditions. J Rheumatol. 1997;24:1734-8.

\section{Submit your next manuscript to BioMed Central and take full advantage of:}

- Convenient online submission

- Thorough peer review

- No space constraints or color figure charges

- Immediate publication on acceptance

- Inclusion in PubMed, CAS, Scopus and Google Scholar

- Research which is freely available for redistribution 